\title{
NITRATO ADDUCTS AND DERIVATIVES: SYNTHESIS AND INFRARED STUDY
}

\author{
MOUHAMADOU BIRAME DIOP ${ }^{* *}$, LIBASSE DIOP ${ }^{1}$ \\ ${ }^{1}$ Université Cheikh Anta Diop, Faculté des Sciences et Techniques, Département de \\ Chimie, Laboratoire de Chimie Minérale et Analytique, Dakar, Sénégal
}

\begin{abstract}
Five new nitrato adducts and derivatives have been synthesized and studied by infrared spectroscopy. The suggested structures are discrete or of infinite chain type, the nitrate behaving as a bidentate or a monodentate ligand, the environment around the tin (IV) centres being octahedral or trigonal bipyramidal. When extra hydrogen bonds are considered supramolecular architectures may be obtained.
\end{abstract}

Keywords: bidentate, monodentate nitrate, discrete and infinite chain structures, octahedral or trigonal bipyramidal environments, supramolecular architectures

\section{INTRODUCTION}

Organo- and halotin (IV) compounds are known for their several applications [1]. Nitrato containing structures have been reported by several groups [2-6]. Seeking for new biologically active compounds, many groups including our have yet published several papers [7-13]. In this dynamic we have, in this work, allowed:

- $\quad \mathrm{SnPh}_{3} \mathrm{Cl}$ to react with $\mathrm{AgNO}_{3}$ and methyl-2-imidazole;

- $\quad \mathrm{SntBu}_{2} \mathrm{Cl}_{2}-\left(\mathrm{tBu}=\right.$ tertiobutyl)- to react with $\mathrm{AgNO}_{3}$;

- $\quad \mathrm{MEIHNO}_{3}-(\mathrm{MEI}=$ methyl-2-imidazole $)$ - to react with $\mathrm{SnBu}_{2}\left(\mathrm{OCH}_{3}\right)_{2}$ or $\mathrm{SnCl}_{2 \cdot 2} \mathrm{H}_{2} \mathrm{O}$.

This has yielded five new compounds, infrared study of which have been carried out then structures suggested on the basis of infrared data.

\section{EXPERIMENTAL SETUP}

$\mathrm{MEIHNO}_{3}$ has been obtained as white crystals on mixing methyl-2-imidazole in water with nitric acid in 1/1 ratio. The compound $\mathrm{A}$ was obtained as a white powder on mixing methanolic solutions of $\mathrm{SnPh}_{3} \mathrm{Cl}, \mathrm{AgNO}_{3}$ and methyl-2-imidazole in 1/1/1 ratio while the compound B was obtained as a white powder on allowing $\mathrm{SntBu}_{2} \mathrm{Cl}_{2}$ to react in ethanol with $\mathrm{AgNO}_{3}$ in $1 / 1$ ratio. The compounds $\mathrm{C}, \mathrm{D}$ and $\mathrm{E}$ were obtained as white powders on allowing $\mathrm{MEIHNO}$ to react with $\mathrm{SnBu}_{2}\left(\mathrm{OCH}_{3}\right)_{2}$ in methanol (C) or $\mathrm{SnCl}_{2} \cdot 2 \mathrm{H}_{2} \mathrm{O}$ in acetonitrile (D and E) respectively in 1/1,2/1 and 1/1 ratio. All the mixtures were stirred around two hours before being submitted to a slow solvent evaporation.

The analytical data [\% calculated (\% found)], have allowed to suggest the following formulae:

A: $\mathrm{SnPh}_{3}\left(\mathrm{NO}_{3}\right)_{2}$.MEIH.SnPh ${ }_{3} \mathrm{Cl} ; \% \mathrm{C}=50.97(50.80), \% \mathrm{H}=3.96(4.09), \% \mathrm{~N}=5.94(5.86)$;

B: $\mathrm{SntBu}_{2} \mathrm{NO}_{3}$ Cl.EtOH.H$\cdot \mathrm{H}_{2} \mathrm{O} ; \% \mathrm{C}=30.45(30.39) ; \% \mathrm{H}=6.64(6.69), \% \mathrm{~N}=3.55(3.60)$;

C: $3 / 2\left(\mathrm{MEIHNO}_{3}\right) \cdot \mathrm{SnBu}_{2}\left(\mathrm{OCH}_{3}\right)_{2} ; \% \mathrm{C}=37.48(37.32), \% \mathrm{H}=6.78(6.71), \% \mathrm{~N}=12.29(12.11)$;

\footnotetext{
* Corresponding author, email: mouhamadoubdiop@ gmail.com

(C) 2017 Alma Mater Publishing House
} 
D: $2\left(\mathrm{MEIHNO}_{3}\right) \cdot \mathrm{SnBu}_{2}\left(\mathrm{OCH}_{3}\right) 2.3 \mathrm{H}_{2} \mathrm{O} ; \% \mathrm{C}=33.82(33.73), \% \mathrm{H}=6.94(7.04), \% \mathrm{~N}=13.15(12.92)$; E: $2 \mathrm{MEIHSnCl}_{5} \cdot \mathrm{MEIHNO}_{3} ; \% \mathrm{C}=15.96(16.05), \% \mathrm{H}=2.34(2.38), \% \mathrm{~N}=10.85(10.91)$.

The elemental analyses have been obtained from "The Microanalyses Laboratory" University of BurgundyDijon-France and from "Laboratoire de Mesures Physiques" Montpellier II University-France. The IR spectra were performed at the University of Burgundy-Dijon-France and at Saint Boniface University-Winnipeg-Canada using a Bruker FTIR spectrometer. IR abbreviations: vs (very strong); s (strong); m (medium), w (weak), sh (shoulder). The chemicals were purchased from Aldrich Company-Germany- without any further purification.

\section{RESULTS AND DISCUSSION}

Let us consider the main IR data (in $\left.\mathrm{cm}^{-1}\right)$ of the studied adducts and derivatives:

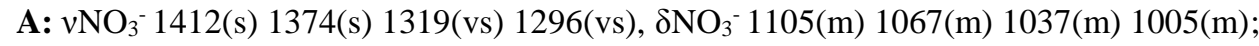

B: $v \mathrm{NO}_{3}{ }^{-} 1435(\mathrm{vs}) 1390(\mathrm{vs}) 1300(\mathrm{vs}), \delta \mathrm{NO}_{3}^{-} 1150(\mathrm{vs}) 1035(\mathrm{~m}) 1010(\mathrm{~m})$;

C: $v_{N^{-}}{ }^{-} 1479(v s) 1425(\mathrm{sh}) 1335(\mathrm{vs}) 1286(\mathrm{vs}), \delta \mathrm{NO}_{3}{ }^{-} 1112(\mathrm{~s}) 1074(\mathrm{~m}) 1041(\mathrm{~m}) 1023(\mathrm{~m})$;

D: $\mathrm{NNO}_{3}{ }^{-1415}$ (vs) 1410(vs) 1380(vs) 1365(vs) 1300(s) 1251(s), $\delta \mathrm{NO}_{3}{ }^{-} 1128(\mathrm{~m}) 1090(\mathrm{w}) 1053(\mathrm{~m})$;

E: $\mathrm{NO}_{3}{ }^{-1} 1410(\mathrm{vs}) 1390(\mathrm{vs}) 1325(\mathrm{vs}) 1290(\mathrm{vs}) 1275(\mathrm{~s}), \delta \mathrm{NO}_{3}^{-} 1120(\mathrm{~m}) 1100(\mathrm{~m}) 1080(\mathrm{~s})$.

From these infrared data, we have suggested for $\mathbf{A}$ a discrete structure in which a nitrate anion acts as a bidentate ligand coordinating a $\mathrm{SnPh}_{3} \mathrm{Cl}$ and a $\mathrm{SnPh}_{3} \mathrm{NO}_{3}$, the environment around the tin centres being trigonal bipyramidal (Figure 1). This type of structure have yet been determined in $\left.\operatorname{Et}_{4} \mathrm{~N}_{2} \mathrm{NO}_{3}\left(\mathrm{SnClPh}_{3}\right)_{2}\left(\mathrm{SnPh}_{3} \mathrm{NO}_{3}\right)\right]$ by Diop et al [4].

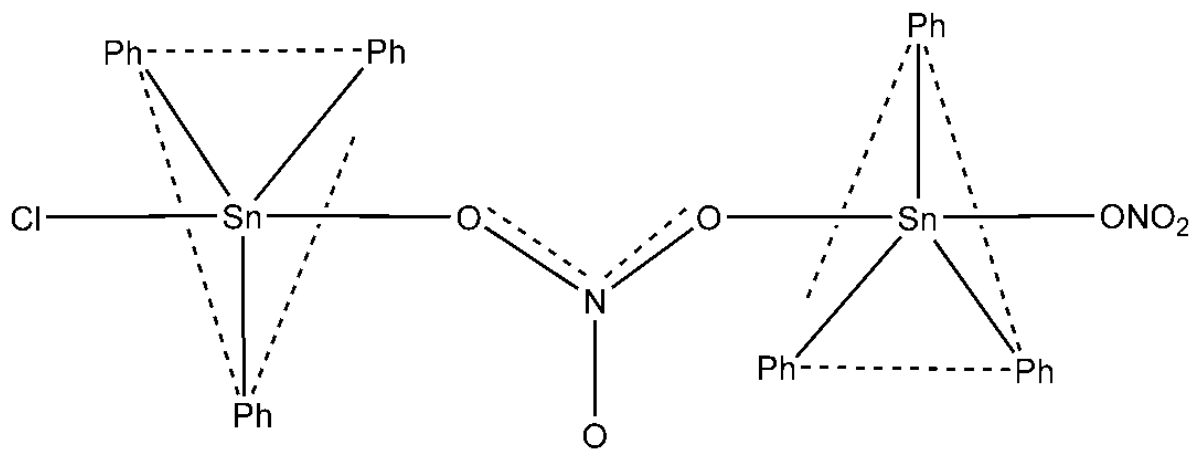

Fig. 1. Proposed structure for the compound A.

We consider $\mathrm{H}_{2} \mathrm{O}$ and $\mathrm{EtOH}$ as ligands. So the proposed structure for $\mathbf{B}$ is discrete with a monocoordinating nitrate, the environment around the tin centre being octahedral (Figure 2a). We can also suggest an infinite chain structure in which the nitrate anions are bidentate bridging the $\left[\mathrm{SntBu}_{2} \mathrm{Cl}\left(\mathrm{OH}_{2}\right) \mathrm{EtOH}\right]^{+}$cations, the coordination number around the tin atoms being 7 (Figure 2b).

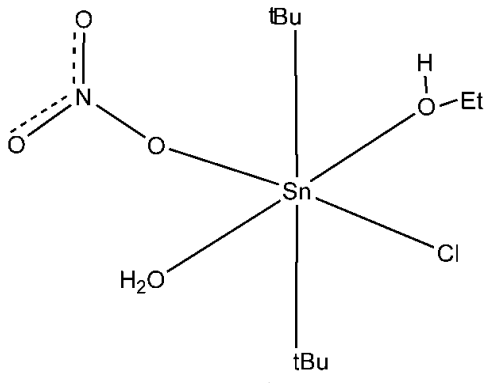

a)

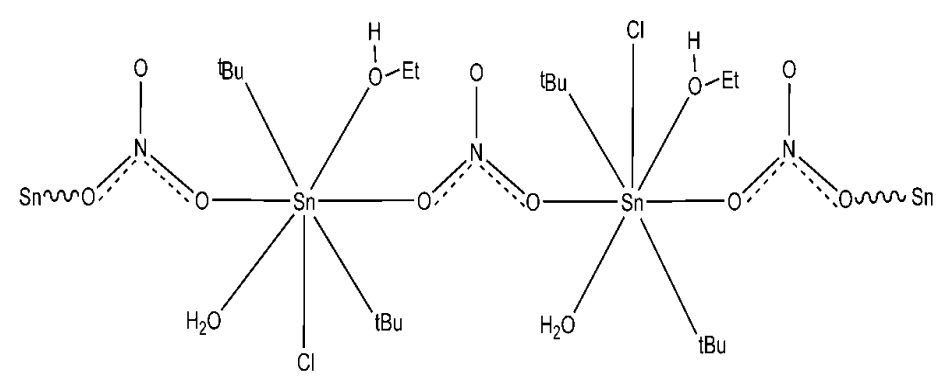

b)

Fig. 2. Proposed structures for the compound B.

For $\mathbf{C}$ we suggest a discrete structure with a central bidentate bridging nitrate and two external monodentate nitrates (Figure 3). 


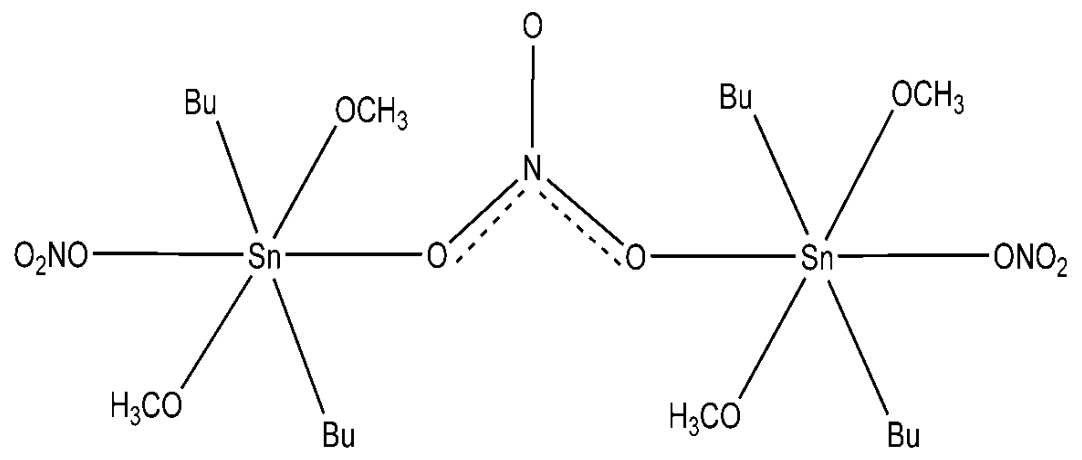

Fig. 3. Proposed structure for the compound $\mathbf{C}$.

For $\mathbf{D}$ a dimer in which the nitrates are monodentate coordinating the $\mathrm{SnBu}_{2}\left(\mathrm{OCH}_{3}\right)_{2}$, the dimerization being ensured by the cations through hydrogen bonds (Figure 4a). We also have suggested an infinite chain structure with monodentate nitrates trans coordinating the $\mathrm{SnBu}_{2}\left(\mathrm{OCH}_{3}\right)_{2}$ molecules, the complex-anions being linked through O----cation----O hydrogen bonds (Figure 4b).

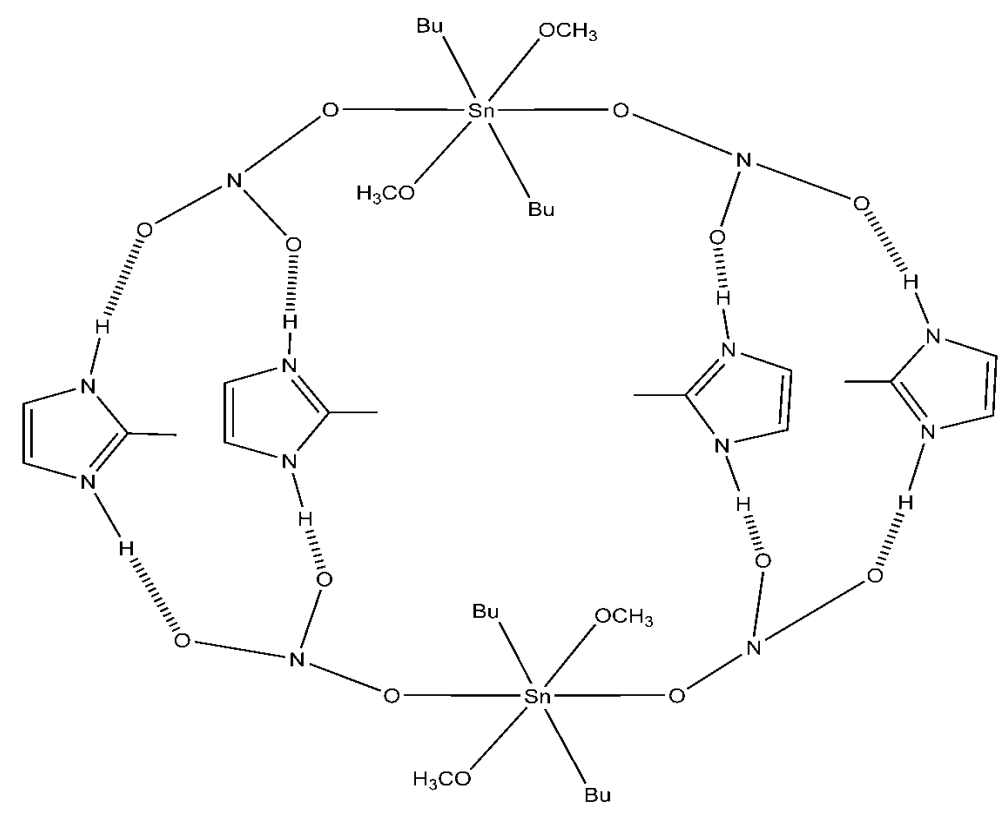

Fig. 4a. Proposed structure for the compound D.

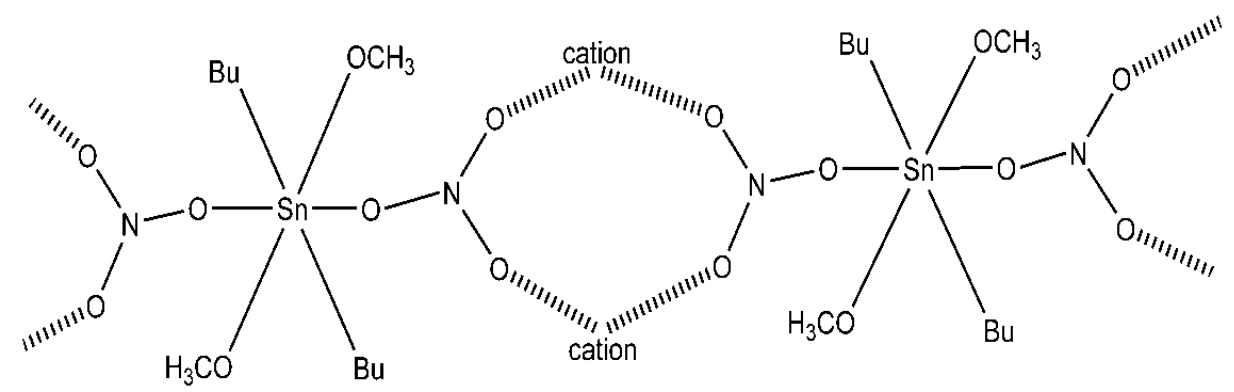

Fig. 4b. Proposed structure for the compound D. 
For $\mathbf{E}$ while considering the complex-anion $\left[\mathrm{NO}_{3}\left(\mathrm{SnCl}_{5}\right)_{2}\right]^{3-}$ we suggest a discrete structure with a bidentate bridging nitrate coordinating two $\mathrm{SnCl}_{5}^{-}$, the environment around the tin atoms being octahedral, the cations may interact through hydrogen bonds (Figure 5).

In all the proposed structures while considering extra hydrogen bonds supramolecular architectures may be obtained.

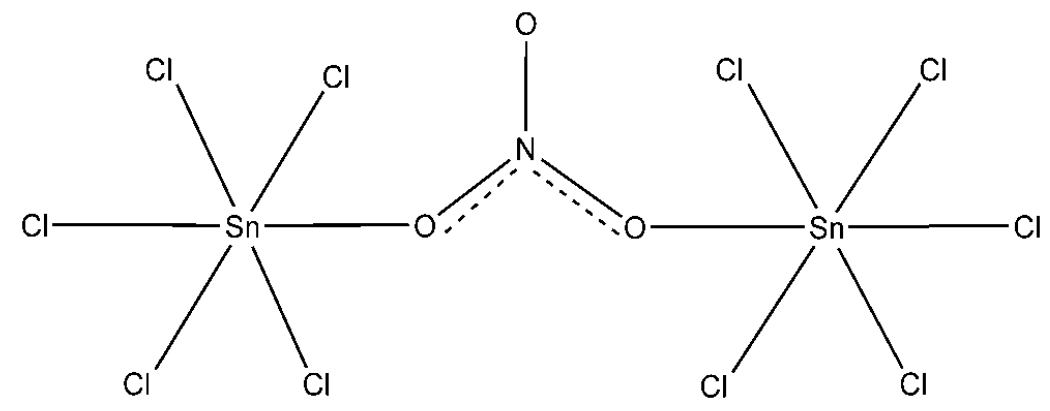

Fig. 5. Proposed structure for the compound E.

\section{CONCLUSIONS}

The studied adducts and derivatives have a discrete - a monomer or a dimer- or an infinite chain structure, the nitrate behaving as a bidentate or a monodentate ligand, the environment around the tin centres being octahedral or trigonal bipyramidal. In all the proposed structures extra hydrogen bonds may occur leading to supramolecular architectures.

\section{ACKNOWLEDGEMENTS}

We thank Professor L. A. DIOP (Collège Universitaire de Saint-Boniface-Winnipeg) and Dr. L. Plasseraud (University of Burgundy-Dijon-France) for equipment support.

\section{REFERENCES}

[1] Evans, J.C., Karpel, S., Organotin compounds in modern technology, Journal of Organometallic Chemistry Library, vol. 16, 1985.

[2] Hou, C., Gan, H-M, Liu, J-C., Crystal structure of catena-poly [[[tetraרaqua $\neg$ zinc(II)]- $\mu-1,4-$-bis-[4-(1Himidazol-1-yl)benzo-yl] piperazine] dinitrate monohydrate], Acta Crystallographica, vol. E71,_2015, p.120-121.

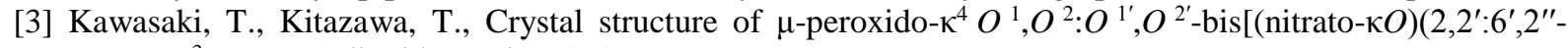
terpyridine- $\left.\kappa^{3} N, N^{\prime}, N^{\prime \prime}\right)$ dioxidouranium(VI)], Acta Crystallographica, vol. E71, 2015, p. 122-123.

[4] Diop, T., Diop, L., Michaud, F., Ardisson, J.D., Et ${ }_{4} \mathrm{~N}\left[\mathrm{NO}_{3}\left(\mathrm{SnClPh}_{3}\right)_{2}\left(\mathrm{SnPh}_{3} \mathrm{NO}_{3}\right)\right]$ : a trinuclear organostannate complex and related derivatives, Main Group Metal Chemistry, vol. 36, no. 3-4, 2013, p. 83-88.

[5] Diop, T., Diop, L., Kucerakova, M., Dusek, M., Acetonyltriphenylphosphonium nitrate, Acta crystallographica, vol. E69, 2013.

[6] Nawaz, S., Tahir, M.N., Nadir, M.A., Mehmoud, B., Ahmad, S.: Crystal structure of bis (thiourea- $\kappa S$ ) bis (triphenylphosphane- $\kappa P$ ) silver (I) nitrate, Acta crystallographica, vol. E71, 2015, p. 220-222.

[7] Allouch, H., Diop, L., Synthesis and infrared study of some new $\mathrm{SnC}_{2} \mathrm{O}_{4} \mathrm{Cl}_{2}$ adducts and complexes Scientific study \& research - chemistry \& chemical engineering, biotechnology, food industry, vol. 13, no. 3, 2012, p. 317-323.

[8] Traoré, B., Diop, L., Sidibé, M,:: Aminophenylarseniato adducts and derivatives of $\mathrm{SnR}_{2} \mathrm{Cl}_{2}(\mathrm{R}=\mathrm{Ph}, \mathrm{Bu})$ : Synthesis and infrared study, Scientific study \& research - chemistry \& chemical engineering, biotechnology, food industry, vol. 13, no. 4, 2012, p. 405-408.

[9] Ndoye, D., Diop, L., 2[(enH $\left.)_{2} \mathrm{O}_{2} \mathrm{C}-\mathrm{SO}_{3}\right] \cdot \mathrm{SnPhCl}_{3}$ andCy $\mathrm{NH}_{2} \mathrm{O}_{2} \mathrm{C}_{-} \mathrm{SO}_{3} \mathrm{H} \cdot \mathrm{SnPhCl}_{3} \cdot \mathrm{H}_{2} \mathrm{O}$ : Synthesis and infrared study, Scientific study \& research - chemistry \& chemical engineering, biotechnology, food industry, vol. 13 , no. 4, 2012, p. 409-412. 
[10] Sarr, M., Diasse-Sarr, A., Diallo, W., Plasseraud, L., Cattey, H., Bis (cyclohexylammonium) tetrachlorido(oxalato) stannate( IV), Acta Crystallographica, vol. E69, no. 8, 2013, p. 473-474.

[11] Gueye, N., Diop, L., Stoeckli-Evans, H., Tetrakis (dipropylammonium) tetrakis (oxalato-к 2 O 1, O 2) stannate (IV) monohydrate: a complex with an eight-coordinate Sn IV atom, Acta Crystallographica, vol. E70, no. 2, 2014, p. 49-50.

[12] Qamar-Kane, H., Fall, A., Diop, L., New $\mathrm{MX}_{2}$ oxalato polynuclear adducts (M=Cd, $\left.\mathrm{Hg}, \mathrm{Zn} ; \mathrm{X}=\mathrm{Cl}, \mathrm{Br}\right)$ : Synthesis and infrared study, Scientific study \& Research chemistry \& chemical engineering, biotechnology, Food industry, vol. 12, no. 4, 2011, p. 357-362.

[13] Sow, Y., Diop, L., Ardisson, J.D., Synthesis, infrared and Mossbauer studies of two new oxalate $\mathrm{SnPh}_{2} \mathrm{Cl}_{2}$ and $\mathrm{SnPh}_{2} \mathrm{Cl}(\mathrm{OH})$ adducts, International journal of biological and chemical sciences, vol. 7, no. 5, 2013, p. 2133 - 2138 . 